\title{
Brachiocephalic Arterial Breach Post-Tracheostomy Innominate Artery Breach after Tracheotomy
}

\author{
A. Elmoqaddem*, N. Mrani Alaoui, T. Cherrad, M. Hmidi, F. Choumi, K. Nadour \\ Anesthesiology and Intensive Care Department, Department of Emergency, Moulay Ismail Military Hospital, Meknes, Morocco \\ Faculty of Medicine, Sidi Mohamed Ben Abdellah University, Fes, Morocco
}

DOI: $\underline{10.36348 / \text { sjmps.2021.v07i01.010 }}$

| Received: 18.10.2020 | Accepted: 04.11.2020 | Published: 16.01.2021

*Corresponding author: Amine Elmoqaddem

\section{Abstract}

Post-tracheostomy brachiocephalic arterial injury (TABC) is a rare complication. We report the case of a 62-year-old patient ho required invasive ventilation through an orotracheal tube for asthma. He had suddenly showed a cataclysmic hemorrhage externalizing around the tracheal cannula with bright red blood. The exploration had revealed a breach of two centimeters at the level of the TABC. Ulceration of TABC after tracheostomy despite its rarity is a fatal complication that must be known by the resuscitators.

Keywords: breach, innominate artery, tracheotomy.

Copyright () 2021 The Author(s): This is an open-access article distributed under the terms of the Creative Commons Attribution 4.0 International License (CC BY-NC 4.0) which permits unrestricted use, distribution, and reproduction in any medium for non-commercial use provided the original author and source are credited.

\section{INTRODUCTION}

Post-tracheostomy brachiocephalic arterial injury (TABC) is a rare complication and potentially a life-threatening with up to $75 \%$ deaths 1$]$, its can begin after several days later [1]

\section{CaSe Report}

We report the case of a 62-year-old patient, known to have asthma, admitted to resuscitation for severe acute asthma, for she was noticed with severe respiratory problems. The patient required invasive ventilation through an orotracheal tube $\left(\mathrm{n}^{\circ} 8\right.$, sterifil). The evolution was marked by the persistence of bronchospasm and the onset recurrent pneumothorax that prompted prolonged ventilation. On the tenth day a tracheostomy cannula (number 8, tracheostomytube ${ }^{\circledR}$ ) was placed in the operating room by an experienced ENT surgeon, under isthmic opposite the fourth tracheal ring. No intraoperative incident was mentioned. On the fourth day of the tracheostomy, the patient had suddenly showed a cataclysmic hemorrhage externalizing around the tracheal cannula with bright red blood, causing cardiovascular collapse without respiratory distress, tracheal suction had not returned blood. Our Immediate Medical intervention reduces bleeding and was achieved by compressing the cannula. The administration of macromolecular solutions, seven globular base and vase- active ingredients had made it possible to restore the hemodynamic situation. The patient had benefited immediately after transclavicular surgery. The exploration had revealed a breach of two centimeters at the level of the TABC. The curative gesture has consisted of a suture of the breach. A tracheostomy cannula ( ${ }^{\circ} 8$, tracheostomytube $\AA$ ) had been put back in place. The rest was simple. Three days later, a recurrence lightning hemorrhagic had occurred causing cardiovascular collapse and respiratory distress with death of the patient in the operating room.

\section{DisCUSSION}

The incidence of erosion of the brachiocephalic arterial trunk following a tracheotomy is in the range of 0.6 to $0.7 \%$ [1]. Among the vascular complications of tracheostomy, the only TABC accounts for $70 \%$ of attacks [2]. Erosion occurs either through direct contact with cannula bent or through the trachea through the balloon or tip of the cannula [1], this is due to the anatomical position of the arterial trunk close to the trachea, thus explaining the frequency of its attack.

The risk factors for the occurrence of this complication are multiple

A Low located tracheostomy, posttracheostomy care (mobilization, hyper extension, hyper flexion), balloon hyperinflation necrosis, mucosal trauma by malposition of the cannula, the use of 
corticosteroids, local infection and malnutrition [1.3]. Prolonged tracheal intubation may also be involved [3].

In tracheostomies patients, the diagnosis of TABC erosion is based on massive bleeding from the opening of the tracheostomy with hemodynamic and respiratory distress. Between $35 \%$ to $50 \%$ of cases experienced minimal bleeding between 2 hours and 4 days before the massive bleeding [4]. Heartbeats in the cannula punctuated by the pulse or bloody tracheal aspirations are warning signs [2]. The initial support consists of inflation or hyperinflation of the balloon to secure the airways and prevent inhalation. The digital compression can limit blood loss. Orotracheal intubation with Balloon hyperinflation may be recommended in patients with continuous bleeding [1, 3] Management of hemorrhagic shock is concomitant. Secondly, the final control of the bleeding occurs in the operating room immediately $[2,3]$. Surveillance should be continues because the risk of recurrence exists [5]. Ulceration of TABC after tracheostomy despite its rarity is a fatal complication that must be known by the resuscitators. The low tracheostomy below the third ring tracheal should be avoided [5] and special attention should be paid to warning signs especially with patients who have the previous risk factors.

\section{CONCLUSION}

Post-tracheostomy brachiocephalic arterial injury (TABC) is a rare complication that can be lifethreatening with up to $75 \%$ mortality, 1 with an onset that can be several days late.

\section{REFERENCES}

1. Narinder, S. (2007). Adrian Fung, Innominate artery hemorrhage following tracheostomy, Otolaryngol Head Neck Surg, 136 (4S): S68-S72.

2. Quinio, P., Foon, J. L. Y., Mouline, J., Braesco, J., \& De Tinteniac, A. (1995, January). Ulcération du tronc brachio-céphalique par une canule de trachéotomie. In Annales francaises d'anesthesie et de reanimation (Vol. 14, No. 3, pp. 296-299). Elsevier Masson.

3. Grant, C. A., Dempsey, G., Harrison, J., \& Jones, T. (2006). Tracheo-innominate artery fistula after percutaneous tracheostomy: three case reports and a clinical review. British journal of anaesthesia, 96(1), 127-131.

4. Jones, J. W., Reynolds, M. A. R. L. E. T. A., Hewitt, R. L., \& Drapanas, T. H. E. O. D. O. R. E. (1976). Tracheo-innominate artery erosion: Successful surgical management of a devastating complication. Annals of surgery, 184(2), 194.

5. Praveen, C. V., \& Martin, A. (2007). A rare case of fatal haemorrhage after tracheostomy. Annals of the Royal College of Surgeons of England, 89(8), W6. 